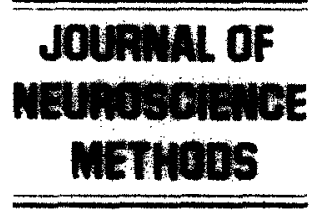

\title{
Microcomputer-based pulse stimulator
}

\author{
F.-S. Jaw ${ }^{*}$, S.-J. Liu, T.-S. Kuo, C.-Y. Wang \\ Center for Biomedical Engineering, College of Medicine, National Taiwan University, Taipei, Taiwan
}

Received 13 May 1995; revised 16 May 1995; accepted 17 May 1995

\begin{abstract}
A low-cost PC-based 2-channel stimulator was designed. This device can generate regular current pulses for many neuroscience experiments. It can also generate irregular pulses for nerve stimulation, thus could possibly avoid the problem of sensitization or habituation in the central nervous system. The stimulation frequency (can be $<0.0005 \mathrm{~Hz}$ ) and pulse duration ( $5 \mu \mathrm{s}$ to $65 \mathrm{~ms}$ ) of this device can be programmed by an interactive user interface. Furthermore, the output current $(0-10 \mathrm{~mA})$ can be continuously varied and was optically isolated to minimize stimulus artifact. Moreover, features such as low-power consumption $(0.2 \mathrm{~mA}$ of a $9 \mathrm{~V}$ battery) and high-compliance output ( $>120 \mathrm{~V}$ ) could accommodate the stimulator for widespread applications. A trial of nerve stimulation was illustrated besides the electrical specifications of the stimulator.
\end{abstract}

Keywords: Pulse generator; Stimulator; PC AT; Constant-current source

\section{Introduction}

Electrical stimulators are widely used in various research, such as functional electrical stimulation (Thrope et al., 1985; Meadows and McNeal, 1989), iontophoretic drug delivery (Park, 1989; Jaw et al., 1995), cardiac pacing (Cochrane et al., 1991), or peripheral nerve locating (Sansome and De Courcy, 1989; Blomfield and Futter, 1991). Conventionally, a single pulse or a train of pulses at a fixed frequency is used for nerve or muscle stimulation. However, owing to the adaptation of the central nervous system (CNS), regular stimulation might cause the problem of sensitization or habituation (Shepherd, 1988). In these cases the evoked responses might become non-linear (Sclabassi et al., 1985). A possible method to overcome this problem is to vary the frequency of stimulation. Such that the nervous system would not be able to 'predict' the timing of stimulation. Stimulators commercially available usually require manual change of the stimulating frequency and are not convenient for this purpose.

To change the stimulation frequency automatically, the stimulator may be controlled by a microcomputer. In principle, there are two kinds of microcomputer-based stimula-

\footnotetext{
* Corresponding author: Tel: 886-2-397-0800 ext. 1453, Fax: 886-2394-0049
}

tors. One is a pattern generator that uses a digital-to-analog converter to generate the analog voltage or current (Caviglia et al., 1983; Roy et al., 1987). The purpose of a pattern or waveform generator is usually for the simulation of biomedical signals (Kohn, 1989) or other special applications such as selective block (Jaw et al., 1991). The other kind is a pulse generator that uses timer or counter circuits to generate the required pulse or train of pulses. This kind of stimulator is commonly used in electrophysiological laboratories.

For neuroscience applications, a stimulator with high compliance constant-current and isolated output is essential (Millar et al., 1994). Also, a short pulse width with a continuously variable amplitude is important for small nerve stimulation. For instance, a $20 \mu$ s pulse with only 5 $\mu \mathrm{A}$ can supramaximally activate all the myelinated fibers in the sacral dorsal roots of the rat (Jaw et al., 1993). In the literature cited, only two stimulators have high-voltage constant-current output (Park, 1989; Millar et al., 1994) and one has photo-isolation (Takahashi et al., 1993). The circuit designed by Park has DC current output due to its iontophoresis purpose. Therefore, it cannot be used for nerve stimulation. The pulse of the Millar design is distorted at duration shorter than $50 \mu \mathrm{s}$ due to the transformer coupling and is thus not suitable for small nerve stimulation. Moreover, these three stimulators are not computer controlled. Some commercially available programmable 
stimulators have the disadvantage of high cost and do not appear to fulfill some of our requirements. So we decided to develop a low-cost and flexible stimulator.

In this paper we describe a PC-based stimulator. The design goals were simplicity, reliability, low cost, and ease of use. This device is for the purpose of programmed nerve stimulation and for other traditional neuroscience applications. The pulse duration ( $5 \mu \mathrm{s}$ to $65 \mathrm{~ms}$ ) and stimulation frequency (can be $<0.0005 \mathrm{~Hz}$ ) can be programmed by an interactive program (that is available upon request). Other features of this device are low-power consumption $(0.2 \mathrm{~mA}$ of a $9 \mathrm{~V}$ battery), high compliance $(>120 \mathrm{~V})$ constant-current $(0-10 \mathrm{~mA})$ output, and optical isolation.

\section{Materials and methods}

\subsection{Stimulator design}

The stimulator design can be separated into digital and analog portions. The digital portion is for the purpose of computer control, whereas the analog portion is for photoisolation and high-voltage constant-current delivery.

Fig. 1 shows the hardware of the digital portion. Basically, it is a timer/counter circuit. Two decoders (LS138) were used to map the I/O address of the 2 timers (8254) from $3 \mathrm{E} 0$ to $3 \mathrm{E} 3$ and from $3 \mathrm{E} 8$ to $3 \mathrm{~EB}$ (in hexadecimal representation). To provide pulse durations of $5 \mu \mathrm{s}$, an 1 $\mathrm{MHz}$ crystal oscillator was used to generate the $1 \mu \mathrm{s}$ time base. There are three 16-bit timers (or counters) in an 8254 integrated circuit (IC). For each channel, an 8254 IC was used. One timer of the IC was used to control the pulse duration from $5 \mu$ s to $65 \mathrm{~ms}$. The other 2 timers of the IC were cascaded to provide stimulus intervals longer than 10 $\mathrm{s}$, since stimulus frequencies higher than $0.1 \mathrm{~Hz}$ may cause the 'wind-up' phenomenon in C-fiber stimulation (Schouenborg, 1984). The second channel of the pulse generator was controlled by the other 8254 IC in the same way and therefore is not shown.

An assembly language driver was developed for controlling the hardware circuit and was linked by the main program, which was written with TURBO $C$ language in DOS environment. This interactive program was developed to facilitate the use of the stimulator. After the pulse. exe command was executed, the control words of the 8254 ICs were programmed. The counter 1 of the 8254 IC was programmed as a hardware retriggerable one-shot

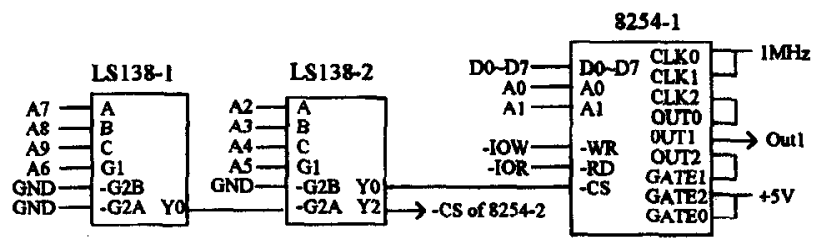

Fig. 1. Digital portion of the stimulator.

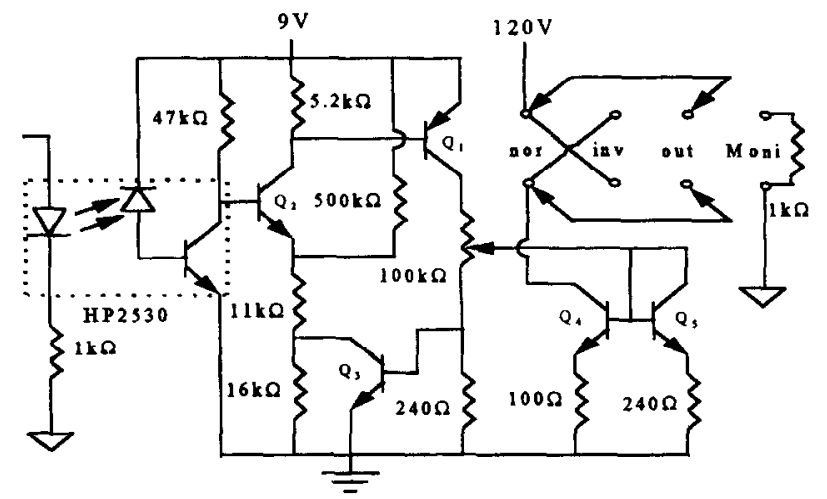

Fig. 2. Analog circuit of the stimulator. The components in the dotted box is the photo-couple (HP2530). All the transistors are npn (2SC1775A) type except the $Q_{1}$ (2SA872A), which is a pnp transistor. The isolated ground can be differentiated with the signal ground in different symbols.

(Mode 1) and the other two cascaded counters were programmed as rate generators (Mode 2). Next, one can input the pulse duration and the stimulating frequency of each channel from the keyboard. Then, the 8254 ICs will be set to generate the pulses automatically without further software overhead.

For artifact reduction, electrical isolation is necessary in stimulator design. Circuits following the isolation barrier should be powered by isolated power supplies. Usually, batteries are used for this purpose. For economic considerations, low-power design is necessary for battery operation. On the other hand, circuits for pulse generation should be high speed. Otherwise, pulses with fast rising and falling edges cannot be obtained. Circuit design with ICs is more convenient than using discrete components. However, the low-power and high-speed requirements cannot be met simultaneously using ICs. Furthermore, a high voltage compliance is not easily achievable with ICs. Therefore, we had to implement the analog portion of the stimulator with discrete components in order to achieve the following aims at the same time: high speed, low power, and high voltage.

The complete circuit of the analog portion is shown in Fig. 2. First, the digital (TTL) pulses were electrically isolated with a photo-coupler. To save battery power, a 47 $\mathrm{k} \Omega$ collector resistor was used to limit the static current of the photo-coupler less than $0.2 \mathrm{~mA}$. However, the output pulses were seriously distorted with such small current. Hence, a Schmitt trigger was used to change the slow rising and falling phases of the distorted pulse into sharp edges. It was composed of three transistors (the $Q_{1}, Q_{2}$, and $\mathrm{Q}_{3}$ ) and consumed only $0.01 \mathrm{~mA}$.

The amplitude of stimulus was not designed to be controlled by the microcomputer due to the following considerations. First, the same size of stimulation is necessary for signal averaging of the evoked central responses. Alternatively, the intensity of stimulus can be changed by the pulse width or by the number of pulses in a train. Therefore, the $100 \mathrm{k} \Omega$ variable resistor at the output of 
Table 1

Some electrical specifications of the stimulator

\begin{tabular}{ll}
\hline$t_{r}$ & $760 \mathrm{~ns}$ \\
$t_{f}$ & $810 \mathrm{~ns}$ \\
$t_{1}$ & $<35 \mathrm{~min}$ \\
$t_{d}$ & $5 \mu \mathrm{s}$ to $65 \mathrm{~ms}$ \\
$R_{o}$ & $570 \mathrm{k} \Omega$ \\
$I_{o}$ & $0-10 \mathrm{~mA}$ \\
$I_{s s}(9 \mathrm{~V})$ & $0.2 \mathrm{~ms}$ \\
$I_{s s}(120 \mathrm{~V})$ & $0 \mathrm{~mA}$ \\
\hline
\end{tabular}

the Schmitt trigger was used to change the output intensity manually. Finally, a current mirror (the $Q_{4}$ and $Q_{5}$ transistors) was used to convert the voltage pulse into constantcurrent output. To achieve the aim of high compliance, the supply voltage of the current mirror was $120 \mathrm{~V}$, which was obtained by cascading 12 popular $9 \mathrm{~V}$ batteries. A switch was used for changing the polarity of the output pulse. The other switch was used for measuring the current intensity of the pulse. The 'Moni' connector was connected to the oscilloscope. While the output was switched to this position, the output current would pass through the $1 \mathrm{k} \Omega$ resistor and the voltage across it could be read from the oscilloscope. Thus, the current intensity of the pulse could be obtained.
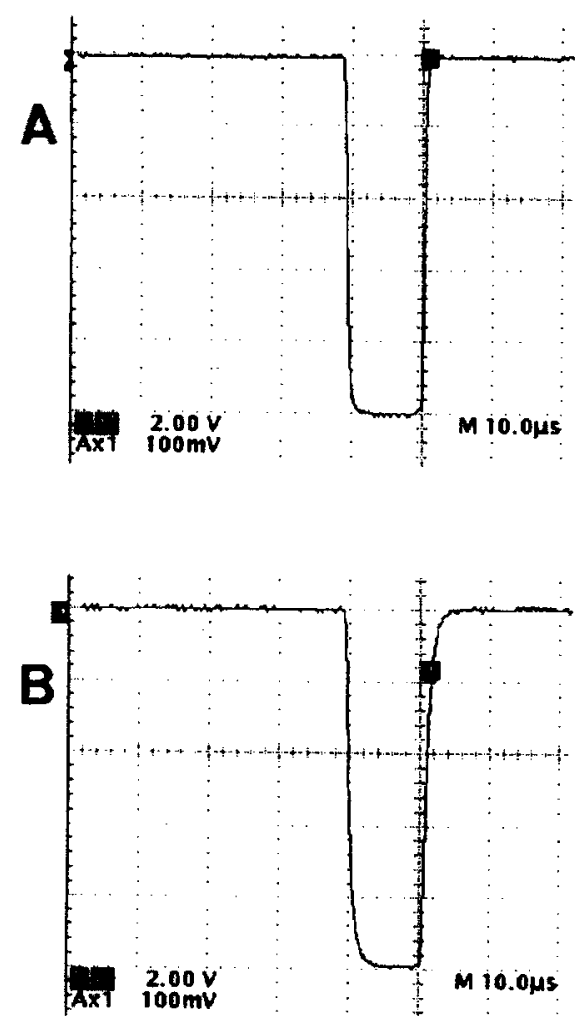
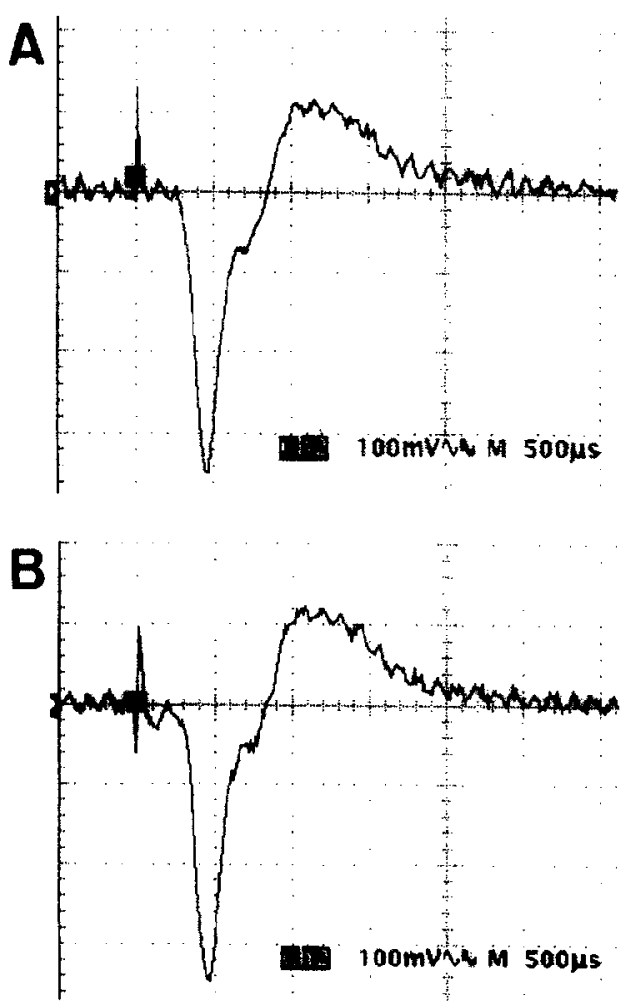

Fig. 4. A: CAP evoked by the DS2 stimulator of the Digitimer. B: CAP evoked by our stimulator.
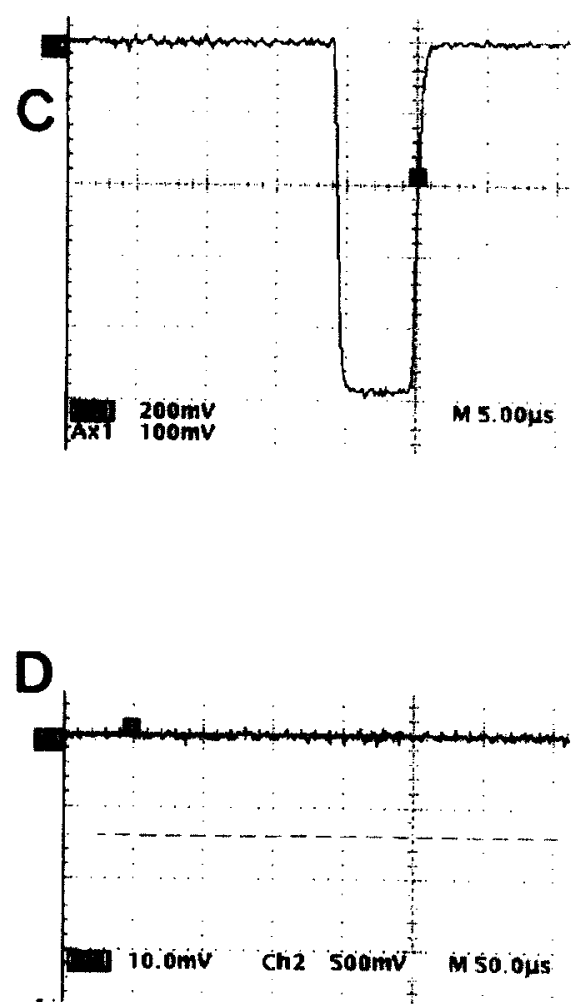

Fig. 3. Output pulses of the stimulator in various test conditions. A: current intensity is $10 \mathrm{~mA}$. B: load is $10 \mathrm{k} \Omega$. C: pulse width is $5 \mu$. D: output current is $0 \mathrm{~mA}$. 


\subsection{Performance measurement}

A $500 \mathrm{MHz}$ digital oscilloscope (Tektronix TDS 520A) was used to measure the electrical characteristics such as rise and fall times of the stimulator. The practical use of the stimulator was compared with a commercial stimulator (Digitimer DS2).

\section{Results}

\subsection{Electrical specifications}

Fig. 3 shows the output pulses with the pulse width, current intensity, and the impedance of load as parameters. The standard test conditions of the output current, pulse width, and load were $1 \mathrm{~mA}, 10 \mu \mathrm{s}$, and $1 \mathrm{k} \Omega$, respectively. For comparison, only one parameter was changed at a time. Other important electrical specifications of the stimulator are summarized in Table 1.

\subsection{Nerve stimulation}

An isolated piece of a dorsal root of a rat was used to evoked compound action potential (CAP) (Jaw et al., 1993). Supramaximal stimulation for A fibers was used to evoke the CAPs shown in Fig. 4.

\section{Discussion}

Because the principles underlying the use of electronic components are not generally known to neuroscientists, complicated and expensive devices are being commonly used. In the present design, only 4 ICs (in the digital interface) and 5 transistors (in the analog portion) were used. Hence, this stimulator is low cost and can be easily constructed. According to the equipment already setup, either the digital or the analog portion can be used independently. For laboratories that have isolation units, the digital portion is needed for programmable capability of a stimulator. On the other hand, the analog circuit might be useful for multichannel stimulators (such as WPI A300 Pulse Master) that usually do not have an isolation unit included.

The proposed stimulator is battery-powered so that most problems of grounding and electrical interference are eliminated. It has been completely tested in electrical characteristics and has been used in nerve stimulation 2 days a week for more than 1 year. The batteries have not been changed yet. Therefore, it is low-power consumption and economic. During the experiment, the stimulating electrodes were usually in contact with the nerve for more than $3 \mathrm{~h}$. We could not find any change of the waveform of the monitored CAP during the experimental period. Thus, there is no DC leakage present on the output of this device.
The 2 CAPs in Fig. 4 show no difference in waveform. Thus, this device is comparable in performance to the Digitimer device. The small pulse width of this stimulator is provided by its fast rise time and fall time characteristics. This feature is important for applications such as determination of the threshold of large fibers in a small nerve. In comparison, the rise time of the ISOLATOR-11 stimulator (Axon Instruments) is $\mathbf{1 0} \mu \mathrm{s}$. Finally, the degree of isolation of a photo-coupler is better than that could be achieved by a transformer, which may depend on special transformers not easily available.

In summary, a small, low cost, and microcomputer-controlled pulse stimulator has been designed. It has been tested both for electrical performance and in practical applications. It is aimed both for the programmable generation of various current pulses and traditional experiments. We hope that this modular and flexible design might be helpful for other neuroscience laboratories.

\section{Acknowledgements}

This work is supported by grants DOH84-HR-310 from NIH of ROC.

\section{References}

Blomfield, D. and Futter, M. (1991) Using a peripheral nerve stimulator as a nerve locator, Anaesth. Intens. Care, 19: 608.

Caviglia, D.D., Gloria, A.D., Donzellini, G., Parodi, G. and Ponta, D. (1983) Design and construction of an arbitrary waveform generator, IEEE Trans. Instrument. Meas., 32: 398-403.

Cochrane, T., Thorne, P.M. and Freeston, I.L. (1991) A microcomputercontrolled cardiac stimulator, J. Med. Eng. Technol., 15: 1-7.

Jaw, F.-S., Yen, C.-T., Tsao, H.W. and Yu, H.J. (1991) A modified triangular pulse stimulator for $\mathrm{C}$-fibers stimulation, J. Neurosci. Methods, 37: 169-172.

Jaw, F.-S., Tsao, H.-W., Yu, H.J. and Yen, C.-T. (1993) Effect of conduction distance on amplitude and area of compound action potentials of A fiber and C fiber, J. Neurosci. Methods, 46: 177-181.

Jaw, F.-S., Wang, C.-Y. and Huang, Y.-Y. (1995) Portable current stimulator for transdermal iontophoretic drug delivery, Med. Eng. Phys., 17: 385-386.

Kohn, A.F. (1989) A versatile waveform generator for testing neuroelectric signal processors, J. Neurosci. Methods, 29: 157-163.

Meadows, P.M. and McNeal, D.R. (1989) A four-channel IBM PC/AT compatible biphasic pulse generator for nerve stimulation, IEEE Trans. Biomed. Eng., 36: 802-804.

Millar, J., Barnett, T.G. and Trout, S.J. (1994) The neurodyne: a simple mains-powered constant-current stimulus isolator, J. Neurosci. Methods, 55, 53-57.

Park, M.R. (1989) Constant current source for iontophoresis, J. Neurosci. Methods, 29: 85-89.

Roy, A., Libsch, F.R. and White, M.H. (1987) A microcomputer-controlled multichannel programmable pattern generator, IEEE Trans. Instrument. Meas., 36: 96-99.

Sansome, A.J. and De Courcy, J.G. (1989) A new dual function nerve stimulator, Anaesthesia, 44: 494-497.

Schouenborg, J. (1984) Functional and topographical properties of field potentials evoked in rat dorsal hom by cutaneous $\mathrm{C}$-fibre stimulation, J. Physiol, 356: 169-192. 
Shepherd, G.M. (1988) Neurobiology, 2nd edn., Oxford University Press, New York, pp. 588-593.

Sclabassi, R.J., Hinman, C.L., Kroin, J.S. and Risch, H.A. (1985) A non-linear analysis of afferent modulatory activity in the cat somatosensory system, Electroenceph. Clin. Neurophysiol., 60: 444454.
Takahashi, M., Rana, A. and Hood, D.A. (1993) Portable electrical stimulator for use in small animals, J. Appl. Physiol., 74: 942-945.

Thrope, G.B., Peckham, P.H. and Crago, P.E. (1985) A computer-controlled multichannel stimulation system for laboratory use in functional neuromuscular stimulation, IEEE Trans. Biomed. Eng., 32: 363-369. 